Estudios Constitucionales, Año 13, No 1, 2015, pp. 465-488.

ISSN 07180195

Centro de Estudios Constitucionales de Chile Universidad de Talca

"El legado de Pablo Dermizaky y su aporte al constitucionalismo boliviano"

Alan E. Vargas Lima

\title{
EL LEGADO DE PABLO DERMIZAKY \\ Y SU APORTE AL CONSTITUCIONALISMO BOLIVIANO
}

(Homenaje Póstumo)

\author{
Alan E. Vargas Lima* \\ Academia Boliviana de Estudios Constitucionales \\ alan.vargas@lapaz.bo
}

"Hay hombres que luchan un dia y son buenos. Hay otros que luchan un año y son mejores. Hay quienes luchan muchos años, y son muy buenos. Pero hay los que luchan toda la vida, esos son los imprescindibles". Bertolt Brecht (Escritor alemán. 1898-1956).

\section{RESUMEN}

El presente estudio bio-bibliográfico, pretende realizar un bosquejo general acerca de la vida, obra y pensamiento del eminente constitucionalista boliviano Dr. Pablo Dermizaky Peredo (†), describiendo algunos de sus rasgos biográficos más importantes, y desarrollando a su vez, una revisión bibliográfica de sus principales obras, con la finalidad de destacar: su loable labor académica de enseñanza del Derecho Constitucional, su indispensable contribución en la consolidación del primer Tribunal Constitucional en Bolivia, y su extraordinario aporte al desarrollo del constitucionalismo boliviano.

\footnotetext{
* Abogado Especialista en Derecho Constitucional y Procedimientos Constitucionales (UMSA). Fue Docente de la Universidad Privada Franz Tamayo y del Centro de Capacitación Municipal. Autor de distintos Libros sobre Derecho Constitucional, Derecho Procesal Constitucional y Derechos Humanos. Columnista de opinión en el Suplemento "La Gaceta Jurídica”, en el Periódico Digital "Oxígeno", y en la página web de "Asuntos del Sur". Escribe para el Anuario de Derecho Constitucional Latinoamericano (Colombia), la Revista Iberoamericana de Derecho Procesal Constitucional (México), y la Revista Iberoamericana de Derecho Administrativo (Argentina). Es miembro de la Academia Boliviana de Estudios Constitucionales, del Instituto Iberoamericano de Derecho Procesal Constitucional-Sección Nacional (Bolivia), y es miembro fundador de la Asociación Boliviana de Derecho Procesal Constitucional. Actualmente, ejerce funciones en calidad de Asesor Legal, dependiente de la Dirección Jurídica del Gobierno Autónomo Municipal de La Paz. Responsable del Blog Jurídico: Tren Fugitivo Boliviano (http://alanvargas4784.blogspot.com/).
} 


\section{NOTA INTRODUCTORIA}

Marzo, se distingue principalmente porque el día 19 se celebra el Día del Padre; sin embargo, este año nos embarga la tristeza de una lamentable noticia, dado que ese mismo día se produjo la inevitable partida, en un viaje sin retorno, de quien fue eminente ensayista, diplomático boliviano, abogado constitucionalista, impulsor y primer Presidente del Tribunal Constitucional de Bolivia, Dr. Pablo Dermizaky Peredo, nacido en la ciudad de Trinidad (Beni) el 24 de marzo de 1923; habiendo cursado sus estudios profesionales en la Facultad de Ciencias Jurídicas y Políticas de la Universidad Mayor de San Simón (Cochabamba), donde fue uno de los alumnos más destacados de su promoción.

Asimismo, en el ejercicio profesional, desempeñó cargos con reconocida solvencia en el ámbito público y privado, habiendo dedicado gran parte de su vida a la cátedra universitaria, dejando una vasta producción bibliográfica de obligada consulta en varias universidades bolivianas y latinoamericanas. Por otro lado, su vida cotidiana, disciplinada, discreta y sin ostentaciones, estuvo llena de valores éticos y morales, destacándose como un hombre muy respetuoso de las personas, sus derechos y libertades, en apego a las reglas y normas preestablecidas para nuestra convivencia pacífica, convirtiéndose así en un digno ejemplo a seguir.

\section{TRAYECTORIA PÚBLICA Y ACADÉMICA}

\section{DE PABLO DERMIZAKY}

La trayectoria de este ilustre profesor es bastante conocida a nivel nacional e internacional, dado que en forma paralela a su excepcional carrera docente, ejerció igualmente algunas funciones públicas y privadas, además de sus constantes actividades académicas, sobre lo cual se puede mencionar lo siguiente:

$\left.1^{\circ}\right)$ En el ámbito público desempeñó funciones diplomáticas como Secretario de la Delegación de Bolivia a la Asamblea General de las Naciones Unidas, en los años 1947, 1948 y 1951; Delegado a la Asamblea General de las Naciones Unidas (1969 y 1970); Primer Secretario de la Embajada de Bolivia en Francia y Encargado de Negocios a.i. (1950 a 1952); Cónsul General de Bolivia en Nueva York (1969 a 1971); Embajador de Bolivia en Bélgica y ante la Comunidad Económica Europea (1979-1980); Delegado de Bolivia a Conferencias Internacionales de la OIT, de la OEA y de la Cuenca de la Plata; Asesor del Ministerio de Relaciones Exteriores de Bolivia (1983-1989). Miembro del Consejo Consultivo Permanente del Ministerio de Relaciones Exteriores (1993 a 1997); funciones importantes que las desempeñó con absoluta solvencia, responsabilidad e idoneidad. 
2o) En el ámbito privado desempeñó el cargo de Asesor Jurídico y Gerente de Relaciones Industriales en la Empresa Manufacturera Boliviana S.A. (MANACO) entre los años 1957-1969; Gerente de la Fábrica de Textiles de Capinota TEXTICA (1971-1972); Gerente General de la Cervecería TAQUIÑA S.A. (1972-1977); Presidente del Directorio de la Empresa Manufactura Boliviana S.A. MANACO (1976-1992).

3o) El Dr. Pablo Dermizaky Peredo fue designado Magistrado Alterno del Tribunal Andino de Justicia (1983-1989); Presidente del Tribunal de Honor del Colegio Departamental de Abogados de Cochabamba (1995-1997); Conjuez de la Corte Superior del Distrito Judicial de Cochabamba (1997-1998).

Como estudioso y publicista del Derecho Constitucional, impulsó de manera decisiva el control jurisdiccional concentrado de constitucionalidad en Bolivia. Desde la cátedra universitaria, y en sus libros, ensayos y artículos (publicados en Revistas Especializadas de España, Francia, Chile y Bolivia), conferencias, seminarios o talleres en los que tuvo ocasión de participar, siempre fundamentó la necesidad de crear un Tribunal Constitucional como el máximo intérprete de la Constitución. En este sentido, participó activamente en el proceso de discusión académica del Proyecto de Ley del Tribunal Constitucional y contribuyó con valiosas ideas, iniciativas y sugerencias ${ }^{1}$.

Como no podía ser de otra manera, el 24 de julio de 1998, previa calificación de méritos, fue designado, por el Congreso Nacional, como Magistrado Titular del Tribunal Constitucional. Una vez tomado posesión del cargo, fue elegido por unanimidad, como Presidente del Tribunal Constitucional, cargo que asumió el 4 de enero de 1999 y lo desempeñó hasta el 15 de febrero de 2001, fecha en la que, por problemas de salud, renunció al cargo de Presidente y Magistrado, privándose al país de uno de los profesionales, juristas y magistrados más probos.

La contribución del Dr. Pablo Dermizaky Peredo en la consolidación del Tribunal Constitucional de Bolivia fue importante, pues junto a sus colegas magistrados trabajó incansablemente en su organización interna, la designación del personal de apoyo, así como en la elaboración y aprobación de sus reglamentos, elaboración del presupuesto y otras actividades. En el ejercicio de la

\footnotetext{
1 Los comentarios y observaciones del autor, al entonces Anteproyecto de Ley del Tribunal Constitucional, pueden consultarse en: Dermizaky Peredo, Pablo. Constitución, Democracia y Derechos Humanos. Sucre, Bolivia: Editorial Judicial, 1999, pp. 61-68.
} 
Presidencia del Tribunal, demostró sus cualidades de hombre probo, líder de equipo, inculcando la mística de trabajo, dentro de los márgenes de seriedad y responsabilidad.

40) En el ámbito académico, fue Catedrático de las materias de Derecho Constitucional, Derecho Administrativo y Ciencias de la Administración en la Facultad de Ciencias Jurídicas y Políticas de la Universidad Mayor de San Simón, entre los años 1972 a 1988. También desempeñó el cargo de profesor de Sociología en la Escuela de Comando y Estado Mayor del Ejército (19751978); y profesor de la materia de Geopolítica de la misma institución (19881990-1993). La labor docente la desempeñó de manera activa, combinándola con la investigación y la difusión del pensamiento constitucional, a través de conferencias y disertaciones en mesas redondas y talleres, en diversas unidades académicas del país.

En el ejercicio de la docencia volcó a favor de los estudiantes, sin mezquindad alguna, la gama completa de sus conocimientos adquiridos con años y años de estudio; es que el Dr. Pablo Dermizaky Peredo fue uno de los pocos constitucionalistas bolivianos que no cultivó la disciplina a mitad de camino, al contrario, hizo del estudio de esta disciplina una de sus grandes pasiones. Fruto de su entrega al estudio y enseñanza del Derecho Constitucional y Administrativo, fue distinguido como Profesor Honorario de la Universidad Boliviana "General José Ballivián" de Trinidad-Beni; Profesor Honorario de la Universidad Mayor de San Simón de Cochabamba, y Profesor Honorario de la UPSA de Santa Cruz; además de haber sido declarado Ciudadano Meritorio de Cochabamba, distinción otorgada por el Gobierno Municipal de Cochabamba; recibiendo la Medalla de Oro al Mérito Profesional, por parte del Colegio de Abogados de Cochabamba. Años más tarde, fue incorporado como Miembro Honorario de la Academia Boliviana de Estudios Constitucionales (ABEC), y también fue miembro correspondiente del Instituto Iberoamericano de Derecho Constitucional (IIDC-México) ${ }^{2}$.

2 Los rasgos biográficos del profesor Pablo Dermizaky, se encuentran detallados en las páginas preliminares del Libro colectivo: Análisis de la Reforma a la Constitución Boliviana, que fue editado hace más de diez años atrás, en homenaje a este destacado constitucionalista boliviano, por parte de la Academia Boliviana de Estudios Constitucionales (Santa Cruz, Bolivia: Editorial El País, 2002). Una breve justificación de este homenaje, ahora puede consultarse en el siguiente sitio web: http://www.abec.org.bo/index.php/ publicaciones/item/21-analisis-de-la-reforma-a-la-constitucion-boliviana. Asimismo, los materiales bibliográficos disponibles en la red internet, correspondientes a algunas de las publicaciones más importantes del profesor Pablo Dermizaky (en el período 1993-2012), pueden consultarse en: http://dialnet.unirioja.es/ 
Sin embargo, en esta oportunidad me interesa rescatar su notable faceta académica, reseñando algunas de sus obras más importantes, para poner de relieve la lucidez de su pensamiento y su indudable trascendencia en el desarrollo y evolución del constitucionalismo boliviano.

\section{APORTE BIBLIOGRÁFICO AL DERECHO ADMINISTRATIVO}

En este sentido, debemos comenzar señalando que la producción bibliográfica del profesor Pablo Dermizaky, empieza en el año 1985, cuando publica la primera edición de su libro sobre "Derecho Administrativo", producto de la experiencia recogida en las aulas universitarias, al ritmo de las exposiciones diarias e inquietudes de sus alumnos. Se trata de una obra, cuyo contenido fue "decantado a través de numerosas consultas de otros textos nacionales y extranjeros, y de revisiones periódicas por las cuales el profesor renovaba constantemente sus lecciones, de manera que los alumnos encontraban siempre algo nuevo y algo mejorado, de un semestre a otro" (Palabras del autor en la introducción de la obra) ${ }^{3}$. Ello, muestra la constante revisión y actualización a que era sometido el texto (que alcanzó su quinta edición en el año 2001) por parte de su autor, quien en la medida de sus posibilidades académicas, no escatimaba esfuerzo alguno para brindar un material de estudio

servlet/autor? codigo $=531357$. Por otro lado, algunos datos sobre la obra literaria de este autor boliviano, se encuentran descritas en el Diccionario Cultural Boliviano: http://elias-blanco.blogspot.com/2011/11/pablodermizaky-peredo.html Entre sus últimas obras, se encuentra precisamente el libro: Justicia Constitucional (La Paz, Bolivia: Editorial Plural, 2010), que recoge seis trabajos escritos por el autor durante los últimos años, algunos de ellos con motivo de sus compromisos académicos dentro y fuera del país, y que están unidos por el hilo conductor de una materia común -la justicia constitucional- que es un elemento esencial de la democracia y del Estado de Derecho. Finalmente, una columna de opinión, dedicada a la memoria del maestro Pablo Dermizaky Peredo, destacando facetas notables de su personalidad, y poniendo de relieve su aporte al constitucionalismo boliviano, fue publicada en el periódico Los Tiempos (Cochabamba, 26 de marzo de 2015), por el Dr. José Antonio Rivera Santivañez, quien fue su discípulo en la Universidad, y colega en el primer Tribunal Constitucional de Bolivia; escrito que se encuentra disponible en el siguiente enlace: http://www.lostiempos.com/diario/opiniones/columnistas/20150326/a-la-memoria-del-maestropablo-dermizaky-peredo_295918_653173.html.

$3 \mathrm{El}$ texto, fue diseñado para la enseñanza-aprendizaje del estudiante universitario y del profesional en Derecho; de ahí que el profesor Dermizaky sostenía que: "La formación del Abogado exige que éste vaya habituándose desde las aulas universitarias al conocimiento, manejo e identificación de las normas concernientes a cada capítulo de la especialidad. Así lo ha remarcado en sus clases el profesor a sus alumnos, para que éstos cobren conciencia de la importancia que ambas partes (doctrina y legislación) tienen en su formación. De ahí que el texto contenga no solamente profusión de citas, sino transcripciones de muchos textos legales pertinentes y en vigencia”. Cfr. Dermizaky Peredo, Pablo. Derecho Administrativo. La Paz, Cochabamba, Bolivia: Editorial Los Amigos del Libro, 1985, pp. 5-6. 
útil, práctico y didáctico -rasgos que caracterizaron toda su obra-, dirigido a los estudiantes universitarios, y también a los profesionales del Derecho.

Entre los temas que conformaban el contenido mínimo de la materia que ofrecía el libro en aquel tiempo, se encuentran: la Ciencia de la Administración, la Administración Pública y Privada, el Derecho Administrativo, la Personalidad de la Administración, la Responsabilidad del Estado y de la Administración, la Organización Administrativa, la Competencia y Jerarquía Administrativas, la Centralización y Descentralización Administrativa en Bolivia, la Organización Administrativa en Bolivia, los Actos Administrativos, los Contratos Administrativos, los Servicios Públicos, la Función Pública, la Carrera Administrativa, el Dominio Público, la Función Jurisdiccional de la Administración, los Recursos, Procesos y Procedimientos Administrativos; temáticas cuyo contenido y desarrollo se encuentran expuestos con bastante claridad y precisión en el texto, que se constituyó en uno de indispensable consulta en varias Universidades bolivianas.

Luego de haberse agotado aquella primera edición, en el año 1988, el autor publicó una segunda edición de la obra, enriqueciendo el contenido de la mayoría de sus capítulos, con base en la bibliografía más reciente que pudo consultar en aquel tiempo (consistente en obras publicadas en España y en varios países de América Latina durante los años 1983 a 1987, según aclara el mismo autor), habiendo agregado al texto, algunos temas referentes a: Gobierno Local o Municipal, Aclaración, Modificación y Extinción de los Actos Administrativos, y el Régimen Disciplinario en la Función Pública, actualizando los distintos capítulos conforme a las reformas de la legislación boliviana hasta ese tiempo.

$Y$ es que según el criterio del profesor Pablo Dermizaky: "El Derecho Administrativo es una disciplina dinámica, sujeta a constante revisión en su doctrina y legislación, por lo mismo que versa sobre la compleja problemática jurídica de la Administración Pública que abarca un campo cada vez mayor"; por lo que, abrigaba la esperanza de que su Manual, llenara las expectativas de los estudiantes de Derecho, que tan buena acogida dispensaron a la primera edición de la obra, y que necesitaban de una clara orientación en una materia tan importante como ésta ${ }^{4}$.

Esta muy didáctica obra del Dr. Dermizaky, que pronto se convirtió en una de indispensable consulta en las Universidades bolivianas, llegó a su ter-

4 Cfr. Dermizaky Peredo, Pablo. Derecho Administrativo. Segunda Edición ampliada y actualizada. La Paz, Cochabamba, Bolivia: Editorial Los Amigos del Libro, 1988, p. 10. 
cera edición en 1997, y una cuarta edición en $1999^{5}$ (con la colaboración de la Editorial Judicial de la ciudad de Sucre), destinada a satisfacer la demanda de profesores y estudiantes, que requieren de un manual donde se conjuga la doctrina administrativa contemporánea con la legislación boliviana que, aunque deficiente, incorpora cada año otras normas sobre la materia, siendo que en ese mismo año se había anunciado la aprobación de una Ley de Procedimientos Administrativos; "pero aún queda mucho camino por recorrer -decía el autor en aquel tiempo-, no existe una ley completa sobre organización de la administración pública, ni otra sobre el proceso contencioso-administrativo, ni sobre actos y contratos administrativos, etc." (sic).

En la quinta y última edición de esta obra del profesor Pablo Dermizaky (publicada el año 2001), se actualizó la mayor parte del texto, incluyendo un nuevo capítulo sobre el Sistema de Regulación Sectorial, que se refiere al denominado Derecho Regulatorio, incorporado en las demás legislaciones del mundo como consecuencia de la Reforma del Estado realizada en las últimas décadas del siglo XX; y es que, según el autor, "Se trata de instituciones (los entes reguladores) esenciales para la ejecución de dicha reforma, que en nuestro país no están funcionando aún como se espera. Faltan ajustes y las normas complementarias que el sistema requiere para mover una maquinaria moderna y compleja" (sic).

De la revisión del texto, se evidencia que esta obra no llegó a incorporar en su contenido, el análisis correspondiente acerca de los alcances y limitaciones (virtudes y defectos) de la Ley No 2341 de Procedimiento Administrativo, promulgada en fecha 23 de abril de 2002. Sin embargo, ello no significa que el autor se hubiera mostrado ajeno a esta reforma en el ordenamiento jurídico administrativo, más al contrario, participó activamente en el debate acerca de la configuración normativa, naturaleza y alcances del entonces Proyecto de Ley de Procedimiento Administrativo que se discutía a nivel del Congreso Nacional ${ }^{6}$.

\footnotetext{
5 En ese mismo año, ya en su calidad de Presidente del Tribunal Constitucional, el Dr. Pablo Dermizaky asistió como expositor al Seminario: "Derecho Administrativo y Sistemas de Regulación”, organizado por la entonces Superintendencia de Recursos Jerárquicos y el Instituto Internacional de Gobernabilidad, en cuyo evento participó con uno de los temas de su especialidad, bajo el interesante título: "La protección jurídica del ciudadano. La defensa del administrado en el derecho constitucional y administrativo". Ensayo que fue publicado en la Revista Internacional de Administración Pública (México), y en el Anuario Iberoamericano de Justicia Constitucional (1999), disponible en: http://dialnet.unirioja.es/descarga/articulo/1976156.pdf.

6 Así por ejemplo, en ocasión de analizar las disposiciones del entonces Anteproyecto de Ley de Procedimiento Administrativo (9 de julio de 1999), el profesor Pablo Dermizaky hizo conocer sus observaciones puntuales en cuanto a la denominación del proyecto, el ámbito de aplicación, el régimen de supletoriedad de otras normas respecto a la falta de disposición expresa, la capacidad del administrado para actuar, la
} 


\section{ENSEÑANZA DEL DERECHO CONSTITUCIONAL}

En la época en que impartía la docencia universitaria, el profesor Pablo Dermizaky también llegó a sistematizar con bastante destreza académica y amplio dominio de la materia, el contenido mínimo de la disciplina del Derecho Constitucional para su enseñanza a nivel universitario en Bolivia, ello con la profunda convicción de que:

"La enseñanza del Derecho Constitucional y de los preceptos constitucionales, es una necesidad vital no sólo para los juristas y para los estudiantes de Derecho, sino para la población en general. Un pueblo que desconoce sus derechos, no puede invocarlos. La educación es, por ello, el principal instrumento de defensa que tiene el hombre común contra la arbitrariedad de los poderosos" (Palabras del autor en la introducción de la obra, que se hallan reproducidas en posteriores ediciones).

Fue así que, en el mismo año de 1985, publicó su Curso de Derecho Constitucional dividido en dos volúmenes (Derecho Constitucional. Tomos I y II. Biblioteca San Simón. Cochabamba, Bolivia: Imprenta Universitaria, 1985 y 1986, respectivamente) ${ }^{7}$, cumpliendo así una responsabilidad académica, en su calidad de Catedrático de Derecho Constitucional y Administrativo de la Facultad de Ciencias Jurídicas y Políticas de la Universidad Mayor de San Simón de Cochabamba, en donde formó varias generaciones de constitucionalistas que desde entonces hasta ahora siguieron sus pasos, transitando por el sendero de la enseñanza, trazado diligentemente por el Maestro.

competencia de los órganos de la Administración Pública, los derechos de los administrados, el silencio de la administración, los actos administrativos, y algunas otras deficiencias formales del proyecto legislativo, todo ello a la luz de la legislación comparada y vigente en ese tiempo. Cfr. Dermizaky Peredo, Pablo. "Breve comentario al Anteproyecto de Ley de Procedimiento Administrativo". En: Revista Opiniones y Análisis. No 44. Anteproyecto de Ley de Procedimiento Administrativo. La Paz, Bolivia: Fundemos y Fundación Hanns Seidel Stiftung, 1999, pp. 29-38.

7 “El curso está separado en dos partes -según justificaba el profesor Dermizaky en la introducción de su obra-, siguiendo la división tradicional de la asignatura en una parte teórica, doctrinal o 'dogmática', que se ocupa de los derechos fundamentales y de las garantías de los mismos; y de otra parte llamada 'orgánica' porque trata de la constitución y funcionamiento de los órganos de gobierno, así como de los regímenes especiales introducidos desde 1938 como aporte del constitucionalismo social que se abrió cauce después de la Primera Guerra Mundial”. En definitiva, se trata de una histórica primera edición, en donde Pablo Dermizaky expresa su agradecimiento a la Universidad cochabambina "en cuyas aulas se formó el autor y donde profesa esta materia y la de Derecho Administrativo desde hace muchos años" (sic); sin embargo, es también una edición agotada, cuyo único ejemplar, solamente me ha sido posible encontrar en la Biblioteca de la Facultad de Ciencias Jurídicas de la Universidad Mayor de San Simón de la ciudad de Cochabamba. 
Cabe agregar que una vez agotada aquella primera edición del Libro -dividido inicialmente en dos tomos no muy extensos-, el autor consideró pertinente publicar una segunda edición de la obra, esta vez condensando los contenidos temáticos de ambos tomos, en un solo volumen (presentación que conservó en las posteriores ediciones); y es que, definitivamente -según aclara el mismo autor- se trató de un nuevo libro "sobre la base del anterior, pero actualizado, enriquecido y concordado con nuevos capitulos, con la legislación secundaria del país y con declaraciones y convenios internacionales" (sic). Hay que destacar que esa visión, ciertamente constituía una forma muy peculiar de enseñar y difundir la disciplina por parte del profesor Pablo Dermizaky en aquel tiempo, siendo que además, en esa nueva edición, el autor vio por conveniente comparar "la constitución vigente con las anteriores a 1967, y con otras de Europa y América, en cuanto concierne a las principales instituciones que rigen en Bolivia y a otras que juzgamos debieran incorporarse a nuestra legislación fundamental".

Es de rescatar su lectura muy clara de la realidad nacional y mundial, acerca del desarrollo de la disciplina hasta ese tiempo, y su estrecha relación con la evolución de los derechos humanos, en el siguiente sentido:

"El Derecho Constitucional reverdece cada día en los predios domésticos y en el campo internacional. Las declaraciones, así como los convenios universales y regionales han dado un gran impulso a esta ciencia que está por convertirse en el marco de las instituciones básicas del siglo veintiuno a nivel mundial. Aunque, por desgracia, están lejos de desaparecer las guerras y la violencia, con su secuela de atropellos a los derechos y a la dignidad del hombre, éste no ceja en su empeño multisecular por establecer un orden internacional y nacional, basado precisamente en el respeto a esos derechos. Como en los primeros tiempos, y aún más que entonces, el hombre lucha contra sus semejantes para desterrar la injusticia, el racismo, la discriminación y el odio que son origen de los males que padece la humanidad. Es reconfortante observar que, en vez de decaer, acrecen los esfuerzos en pro de ese ideal mientras arrecia la embestida del armamentismo y su cortejo, la violencia, la miseria y la opresión" (Palabras del autor, en el prólogo de la segunda edición de su obra, publicada el año 1991).

En esta obra (que ha cumplido 30 años de vigencia desde su primera edición, y gracias a su amplia acogida llegó a su décima edición actualizada en el año 2011) $)^{8}$, el profesor Pablo Dermizaky sostenía que el Derecho Constitucional, "es

8 Cfr. Dermizaky Peredo, Pablo. Derecho Constitucional. Décima Edición revisada y concordada con la Constitución vigente y las leyes de desarrollo constitucional. Cochabamba, Bolivia: Grupo Editorial Kipus, 2011. Entre las diversas reacciones, favorables y contrarias, que provocó la aprobación del proyecto 
una rama del Derecho Público Interno que determina (léase estudia) la organización jurídica y política del Estado, asi como los derechos y deberes de los ciudadanos"; vale decir, que se trata de una ciencia fundamental -según criterio del autor-, a la cual confluyen y se subordinan todas las ramas del derecho público y privado, ello siguiendo el criterio de Kelsen, quien decía que constituye el fundamento de todo el derecho restante.

En cuanto a su naturaleza, el autor precisaba que esta disciplina: "Es una materia esencialmente jurídica porque contiene preceptos de aplicación obligatoria, y formalmente politica porque se refiere a las instituciones de éste género que se relacionan con el Estado" ". Cabe anotar que la breve definición antes descrita, justifica su sencillez en los destinatarios de la obra, que fueron precisamente los estudiantes universitarios (de primer o segundo año de carrera) que recién se introducían al estudio de la disciplina, con absoluta incertidumbre acerca de su naturaleza jurídica, su contenido y alcances ${ }^{10}$.

de Constitución en el año 2009, y en plena víspera de su aprobación mediante referendo popular, resulta interesante encontrar la opinión del Dr. Pablo Dermizaky, quien expresaba su profunda preocupación y descontento frente al texto definitivo de aquel proyecto, con las siguientes palabras: "La criatura que se tratará de bautizar el 25 de enero próximo, ha nacido con defectos incurables de ilegalidad, con malformaciones elefantiásicas en su redacción y con impropiedades de conceptos que la desfiguran totalmente. El constitucionalismo contemporáneo se basa en la Constitución normativa como ley fundamental del orden jurídico. Normativa significa que todos los preceptos, principios y valores contenidos en la Constitución, y que forman el "bloque de constitucionalidad", son directamente aplicables, sin necesidad de reglamentación previa, y exigibles ante la justicia. (...) El proyecto de Constitución (a ser sometido a votación el próximo 25 de enero) es un anacronismo porque la mayoría de sus cláusulas son programáticas, es decir meramente declarativas, propias de un programa electorero y no de una ley fundamental. Véanse, a propósito, los artículos 16, 18, 19, 20, 33, 35-I, 37, 41, 54, 67, 267 y 313, relativos a los derechos expectaticios sobre salud, educación, vivienda, medio ambiente, erradicación de la pobreza, reivindicación marítima, entre algunos. El texto es ampuloso y rebuscado. Hay repeticiones innecesarias y contradicciones flagrantes. Se repite los caracteres de la educación en los artículos 78.II y 91.II, como ejemplo”. Pablo DermizakY. "Muchas cláusulas son declarativas". Análisis publicado en el periódico Los Tiempos (20 de enero de 2009). Disponible en: http://www.lostiempos.com/diario/actualidad/nacional/20090120/muchas-clasulas-sondeclarativas_22553_35411.html.

9 Cfr. Dermizaky Peredo, Pablo. Derecho Constitucional. Tercera Edición ampliada y actualizada. Cochabamba, Bolivia: Editorial Serrano, 1996, pp. 20-21.

10 "El concepto de Derecho Constitucional -decía Dermizaky- fluye, naturalmente, del de Constitución, aunque bien es cierto que lo rebasa porque, como observa Linares Quintana, esta disciplina se ocupa no sólo de la normativa, sino de las instituciones propias del Estado. Por ello se dice que el Derecho Constitucional es esencialmente jurídico (normativo) y formalmente político (institucional)...”. Cfr. Dermizaky Peredo, Pablo. Justicia Constitucional y Estado de Derecho. Segunda Edición. Cochabamba, Bolivia: Editorial AleXander, 2005. Pág. 178. 
Asimismo, conviene recordar que una de las fuentes de esta disciplina, y la más importante, es precisamente la Constitución ${ }^{11}$, sobre la cual, el profesor Pablo Dermizaky explicaba su significado, señalando que: "Una Constitución es el código, norma o ley fundamental de un pais, que determina la estructura jurídicopolitica del Estado, la forma o sistema de su gobierno y los derechos y deberes de la población", y agregaba además que: "como toda norma legal, la Constitución no es un fin en sí, sino un medio para conseguir el equilibrio entre gobernantes $y$ gobernados, fijando limites y controles al poder de los primeros, y derechos y obligaciones para los segundos"12.

Esta concepción, describe el contenido mínimo de toda Constitución, en consonancia con el postulado liberal expuesto en el artículo 16 de la Déclaration des droits de l'homme et du citoyen (1789), que estableció expresamente lo siguiente: "Una sociedad en la que no esté asegurada la garantía de los derechos ni reconocida la división de poderes, no tiene Constitución”. Esta declaración -decía Dermizaky en su obra-, coincidente con la primera Constitución escrita (la de Estados Unidos de Norteamérica de 1787), precisa el significado moderno del vocablo Constitución, en el sentido de norma fundamental que establece la organización del gobierno en órganos separados que impiden la concentración del poder en uno solo de ellos, lo que garantiza los derechos fundamentales de las personas.

Esta idea de Constitución, plasmada en la Declaración de Derechos del Hombre $y$ del Ciudadano, nos ayuda a comprender la estructura básica que debe poseer toda Constitución, dividida clásicamente en dos partes: dogmática y orgánica. Sin embargo, cabe considerar que según la doctrina constitucional contemporánea, la Constitución contiene diversas clases de normas, como las axiológicas (que consagran los valores supremos y principios fundamentales), dogmáticas (que proclaman los derechos fundamentales y garantías constitucionales), y las orgánicas (que regulan la organización del Estado y el ejercicio del Poder Público); de ahí

11 "La Constitución -decía Pablo Dermizaky- es el silabario de la ciudadanía. En ella debe aprender el hombre las primeras letras de su naturaleza política y social; sus derechos, sus deberes, las posibilidades y limitaciones que moldean su actividad en una sociedad organizada. De ahí que la Constitución debe ser enseñada y divulgada en todas partes, en la escuela y en el hogar, en el gobierno y en la Universidad, en la empresa y en el taller, en los colegios profesionales y en el foro sindical. La Constitución es el catecismo de la nacionalidad, el breviario del honor, el oráculo de la verdad”. Cfr. Prólogo de Pablo Dermizaky Peredo, al libro de: Rivera S., José Antonio. Reformas a la Constitución: ¿Modernización del Estado? Cochabamba, Bolivia: Editorial Kipus, 1994.

12 Cfr. Dermizaky Peredo, Pablo. Derecho Constitucional, pp. 47 y 49. 
que, actualmente la mayoría de las Constituciones latinoamericanas se caracterizan por hallarse precedidas de una parte axiológica donde se establecen los principios fundamentales y valores supremos (principios y valores ético-morales, en el caso de Bolivia) que son transversales a todo el ordenamiento jurídico ${ }^{13}$.

\section{Estudio de la Constitución Política del Estado}

Cabe destacar también que en el año 1987, el profesor Dermizaky, publicó una versión comentada y concordada de la Constitución Política del Estado de 1967 (que hasta ese tiempo había cumplido veinte años de vigencia en Bolivia), con el propósito de llenar un vacío en la bibliografía jurídica boliviana, dado que hasta ese tiempo no existía un texto de la Constitución Política concordado con la propia Constitución y con la legislación secundaria, así como con las principales Declaraciones Internacionales de Derechos que constituyen parte esencial del Derecho Constitucional codificado de nuestro tiempo. En

13 De ahí que, la Constitución se puede definir como un pacto social y político adoptado por el pueblo, en el cual se determina el sistema constitucional del Estado, estableciendo las reglas básicas para lograr una convivencia pacífica y la construcción de una sociedad democrática, basada en los valores supremos, como ideales que una comunidad decide constituir como sus máximos objetivos a desarrollar por el ordenamiento jurídico y expresarlos en su estructura social-económica-política; los principios fundamentales, como los presupuestos lógicos y líneas rectoras o básicas del sistema constitucional que orientan la política interna y externa del Estado; así como en los derechos fundamentales y garantías constitucionales de las personas, cuya garantía de cumplimiento y observancia, constituye uno de los fines y funciones esenciales del Estado Plurinacional, que además tiene el deber de promoverlos, protegerlos y respetarlos. Cfr. Rivera S., José Antonio; Jost, Stefan y otros. La Constitución Política del Estado. Comentario Crítico. Tercera Edición, actualizada con las reformas del 2004. Cochabamba (Bolivia): Talleres Gráficos Kipus, 2005, p. 38. Por su parte el Tribunal Constitucional de Bolivia, en la Sentencia Constitucional No 0773/2005-R, de fecha 7 de julio de 2005, ha establecido que los principios fundamentales ocupan un lugar preponderante en nuestro ordenamiento constitucional: "Estos principios hacen referencia a las normas que fundamentan todo el sistema constitucional y tienen por objeto determinar los rasgos esenciales del sistema político, la titularidad del poder, la modalidad de su ejercicio, así como su finalidad. Estos principios constituyen verdaderos mandatos jurídicos, dirigidos, en primer término, al legislador -y también al órgano ejecutivo, cuando asume su facultad reglamentaria-, para que sean tomados en cuenta en el proceso de creación de la normas, pues al ser éstos la base en la que se inspira el modelo de sociedad que la Constitución propugna, debe existir armonía entre la ley a crearse y los principios constitucionales. En segundo término, los principios, como mandatos jurídicos, también se dirigen a las autoridades judiciales o administrativas que van a aplicar las normas jurídicas, en el entendido que al ser jerárquicamente superiores, presiden la interpretación de todo el ordenamiento, e inclusive de la Constitución misma. Las funciones anotadas, coinciden con el carácter informador del ordenamiento jurídico, que tienen los principios; carácter que, de acuerdo a la doctrina, implica que estos principios son directrices para la elaboración de las leyes y para la labor interpretativa, además de ser un parámetro para determinar la inconstitucionalidad de las normas $(\ldots)$ ". 
ese propósito, esta edición comentada de la Constitución "contiene un estudio general sobre la Constitución boliviana, sus concordancias, notas y comentarios que han sido sugeridos al autor a través de la enseñanza de la cátedra y de la lectura del Derecho Constitucional comparado, asi como el texto de otras Constituciones. Las notas y comentarios son breves, como corresponde al propósito enunciado, pues los estudiosos encontrarán un desarrollo más amplio de los temas, en la obra del propio autor..." (sic).

Esta versión comentada de la Constitución, incluye un estudio preliminar sobre la naturaleza y contenido de la Constitución, algunos antecedentes de la Constitución boliviana -haciendo referencia a las características principales de la Constitución Bolivariana y sus fuentes de inspiración-, para luego precisar la estructura de la Constitución vigente en aquel tiempo (promulgada el 2 de febrero de 1967), observando el autor, que a diferencia de la mayoría de las Constituciones, "la Constitución boliviana no está precedida de un preámbulo que condense sus postulados esenciales, como ocurre en la mayoría de las Constituciones de Europa $y$ de América" (sic), al tiempo que describía los principios del constitucionalismo liberal y social, que inspiraron sus diferentes disposiciones.

Ante la favorable acogida que tuvo aquella primera edición del texto constitucional, el profesor Dermizaky publicó en el año 1992, una segunda edición de los comentarios y concordancias de la Constitución vigente en ese tiempo, incluyendo esta vez, una propuesta de reformas a la Constitución Política del Estado ${ }^{14}$.

En dicho trabajo, a modo de adherirse a los diversos sectores de la población que exigían una profunda reforma constitucional, planteó por vez primera, la

\footnotetext{
14 Esta propuesta integral de reformas, ya había sido presentada con anterioridad por el mismo autor, en ocasión del primer Seminario sobre "Reformas Constitucionales", llevado a cabo los días 31 de octubre y $1^{\circ}$ de noviembre de 1991, en la ciudad de Santa Cruz, con los auspicios de la Directiva de la H. Cámara de Diputados y de su Comisión de Constitución, Justicia y Policía Judicial. En su contenido, desarrolló diversos temas que en su criterio eran esenciales y necesarios de modificar o incorporar a la Constitución, como por ejemplo: Preámbulo, Símbolos, idioma y territorio, Estado Plurinacional, Responsabilidad del Estado, Derecho Internacional, Derechos Fundamentales, Derechos no mencionados en la Constitución, Gobiernos usurpadores, Ciudadanía, Ampliación de los Derechos políticos (proponiendo la incorporación de: la iniciativa popular, el referéndum, la revocatoria, las candidaturas cívicas o agrupaciones ciudadanas), Órgano Legislativo, Órgano Ejecutivo, Régimen Interior, Poder Judicial, Control de Constitucionalidad, Ministerio Público, Contraloría General de la República, Recursos Naturales, Medioambiente y Ecología, Gobiernos Municipales, Conservación del orden público, Procedimiento para reformar la Constitución, además de algunas correcciones de forma y otras medidas indispensables. Cfr. Honorable CÁmara DE Diputados-Comisión de Constitución, Justicia y Policía Judicial. La Reforma Constitucional. La Paz (Bolivia): Empresa Editora Proinsa, 1992, pp. 55-82.
} 
siguiente sugerencia: "Debe incluirse un articulo que reconozca oficialmente que Bolivia es un Estado Plurinacional y pluricultural; que protege la existencia, idiomas, cultura y desarrollo de las nacionalidades asentadas en su territorio, y que promueve su integración efectiva con el resto de la población". Esta propuesta, muestra la lectura muy objetiva de la realidad boliviana, por parte del autor, en cuanto a su composición social eminentemente plural y diversa, a cuyo efecto, también ponía de relieve la necesidad de proteger y preservar la cultura de los pueblos indígena originario campesinos, cuyos idiomas y cosmovisiones, actualmente han alcanzado un justo reconocimiento constitucional (a través de la Constitución aprobada el año 2009).

\section{CONSOLIDACIÓN DEL PRIMER TRIBUNAL CONSTITUCIONAL}

Por otro lado, haciendo referencia a los alcances muy restringidos que hasta la década de los años 90's tenía el control de constitucionalidad en la legislación boliviana, en su propuesta de reforma constitucional, el profesor Dermizaky observaba también lo siguiente: “(...) el control se ejerce a instancia de parte solamente, y no de oficio, y la declaración de inconstitucionalidad por la Corte Suprema de Justicia entraña la inaplicabilidad de la norma solamente en el caso concreto cuestionado, y no la abroga ni tiene efecto erga-omnes"; por ello, se constituye en uno de los primeros juristas bolivianos, que con bastante sabiduría planteó la necesidad de incorporar un Tribunal Constitucional en Bolivia, que se encargue del control de constitucionalidad de las leyes, a cuyo efecto, con base en el amplio bagaje de conocimientos que poseía sobre la materia, el autor argumentaba que:

"Esta materia es la base del orden jurídico nacional y, por lo tanto, debe confiarse a un tribunal especial que se ocupe de examinar, de oficio, la legalidad de las leyes, decretos y resoluciones, antes de su aprobación (lo que no ocurre actualmente en Bolivia); que declare de oficio la inconstitucionalidad de leyes, decretos y otras normas vigentes; que falle en las demandas, a instancia de parte, sobre inconstitucionalidad de las mismas normas; que conozca, de oficio o a denuncia, sobre la ilegitimidad de los nombramientos de altos funcionarios de Estado, etc." 15 .

15 Cfr. Constitución Política del Estado (Con Introducción, Notas, Comentarios y Concordancias por el Dr. Pablo Dermizaky Peredo). Segunda Edición. Cochabamba-La Paz, Bolivia: Editorial Los Amigos del Libro, 1992, p. 39. Años más tarde, en una conferencia pronunciada por el autor en la ciudad de Cochabamba (1996), y a tiempo de referirse al entonces Anteproyecto de Ley del Tribunal Constitucional, hizo conocer su opinión señalando que: "La importancia de la jurisdicción constitucional coloca al Tribunal Constitucional en la cúspide de la pirámide institucional del país. De su labor dependerá en 
Posteriormente, y haciéndose eco de ésta y otras propuestas que surgían de los principales actores de la sociedad boliviana, en 1993 el entonces Congreso Nacional emitió la ley No 1473 que declaró la necesidad de reformar la Constitución Boliviana, proponiendo entre otros aspectos, la incorporación de tres instituciones esenciales para consolidar la democracia y proteger los derechos de las personas: El Defensor del Pueblo, El Tribunal Constitucional, y el Consejo de la Judicatura, mismas que fueron incorporadas como parte de la institucionalidad boliviana, a través de la ley $N^{o} 1585$ de Reforma a la Constitución Política del Estado, de fecha 12 de agosto de $1994^{16}$, que introdujo diversas modificaciones al texto constitucional que hasta ese entonces, se hallaba vigente sin cambio alguno desde el año 1967.

A ello se debe agregar, que dicha reforma constitucional indudablemente se constituye en un hecho trascendental con una importancia histórica sin precedentes dentro del sistema constitucional, puesto que en aquella oportunidad,

gran medida, el desarrollo positivo de la vida pública y privada, por lo que, debe tener la confianza de gobernantes y gobernados por igual. Lamentablemente, el marco constitucional no constituye base sólida para su organización y funcionamiento. Quienes participamos de alguna manera en su alumbramiento, tenemos la responsabilidad histórica de llamar la atención sobre esta circunstancia, para que la criatura no nazca lisiada, incapaz de cumplir su misión”. Cfr. Dermizaky Peredo, Pablo. Constitución, Democracia y Derechos Humanos, p. 67.

16 Mediante dicha reforma constitucional, se modificaron aproximadamente treinta y cinco artículos de la Constitución boliviana, cuyo alcance puede resumirse en lo siguiente: a) Inserción de los pueblos originarios y comunidades indígenas a la estructura social del Estado, a cuyo efecto se definió la estructura social como multiétnica y pluricultural, así como de las bases de su organización como son la unión y la solidaridad; b) Modificaciones en el sistema electoral, cambiando el requisito de la edad para el ejercicio de la ciudadanía de 21 años que regía hasta entonces, a 18 años; c) Fortalecimiento del entonces Poder Legislativo, a través de la reforma del sistema de elección de los Diputados para devolverles legitimidad y representatividad, con la introducción del sistema del doble voto en circunscripciones uni y plurinominales; d) Estabilidad y gobernabilidad en el ejercicio del gobierno nacional, reformando las reglas para la elección congresal del Presidente y Vicepresidente de la República, en aquellos casos en los que ninguno de los candidatos obtenga mayoría absoluta de votos en las elecciones generales; e) Plena vigencia y protección de los derechos humanos como base del orden democrático constitucional, a cuyo efecto se ha creado la Defensoría del Pueblo con la misión de velar por la vigencia y cumplimiento de los derechos y garantías de las personas con relación al sector público; f) Fortalecimiento de los gobiernos locales autónomos como unidades básicas del desarrollo sostenible sobre la base de la planificación participativa y el control social; g) Seguridad jurídica y fortalecimiento del orden constitucional reformando la estructura del entonces Poder Judicial (actual Órgano Judicial), mediante la creación del Tribunal Constitucional como máximo intérprete jurisdiccional de la Constitución, asignándole la función del control concentrado de constitucionalidad. Cfr. RIVERA SAntivañEz, José Antonio. Reforma Constitucional en Democracia. En: Análisis de la Reforma a la Constitución Boliviana, editado por la Academia Boliviana de Estudios Constitucionales. Santa Cruz-Bolivia: Editorial El País, noviembre de 2002, pp. 5-8. 
y por vez primera en la historia republicana de nuestro país, se reformó la Ley Fundamental dando cumplimiento a los mecanismos y procedimientos especialmente previstos al efecto por la propia Constitución, vale decir que se aprobó, sancionó y promulgó la Ley de Reforma Constitucional en observancia de las normas previstas por los artículos 230 al 232 del texto constitucional vigente en ese tiempo.

No obstante la vigencia de la Constitución reformada, el Tribunal Constitucional de Bolivia no ingresó en funciones sino hasta julio de 1998, cuando el entonces Congreso Nacional, previa calificación de méritos, designó a los Magistrados del primer Tribunal Constitucional de Bolivia, que se instaló formalmente el 5 de agosto de 1998, e inició sus labores jurisdiccionales el $1^{\circ}$ de junio de 1999 (luego de su "vacatio legis"), con el respaldo de la ley No 1.836 de $1^{\circ}$ de abril de 1998, emitida para regular su estructura, organización y funcionamiento.

Fue en ese tiempo, que los miembros del flamante Tribunal Constitucional (René Baldivieso Guzmán, Hugo de la Rocha Navarro, Willman Durán Ribera y Elizabeth Iñiguez de Salinas), por voto unánime eligieron al Dr. Pablo Dermizaky Peredo, para que asumiera la presidencia de dicha institución; cargo que asumió el 4 de enero de 1999 y lo desempeñó con dignidad hasta el 15 de febrero de 2001, fecha en la cual, por problemas de salud, renunció al cargo de Presidente y Magistrado, privándose al país de uno de los profesionales, juristas y magistrados más probos de los últimos tiempos.

El Dr. José Antonio Rivera Santivañez -que se desempeñó como Magistrado suplente durante la primera época del Tribunal Constitucional de Bolivia-, en su reciente columna de opinión dedicada a la memoria del Maestro, brinda un testimonio de la faceta de Juez Constitucional del Dr. Pablo Dermizaky, con las siguientes palabras:

"En el ámbito jurisdiccional no sólo que desempeñó con sobrada solvencia profesional la labor de Magistrado del Tribunal Constitucional de Bolivia, sino que fue la pieza clave para la configuración y puesta en funcionamiento de dicho órgano constitucional; pues en su condición de primer Presidente de aquella institución, junto al resto de los magistrados titulares y suplentes, equipo del que tuve el honor de formar parte, trabajó de manera denodada y silenciosa para organizarlo administrativa y jurisdiccionalmente; con un presupuesto limitado y prescindiendo de asesores o consultores, los 10 magistrados, encabezados por Dermizaky, elaboraron los reglamentos, alquilaron un inmueble y lo equiparon; seleccionaron y contrataron al personal de apoyo jurisdiccional y administrativo, para luego tramitar una ley que, modificando la vacatio legis prevista por la Ley No 1.836, posibilitó adelantar el inicio de las funciones jurisdiccionales del Tribunal Constitucional. 
En el tiempo que presidió al Tribunal Constitucional le dio su sello personal de transparencia, mística de trabajo y responsabilidad; cuidó celosamente que el Tribunal no incurriera en retardación de justicia controlando personalmente el despacho de las causas; defendió con firmeza y convicción la independencia del Tribunal y los magistrados; pues no dudó un segundo en rechazar una convocatoria efectuada por el Presidente del Congreso Nacional, de ese entonces, a una reunión con los Vocales de la Corte Nacional Electoral que enfrentaba ante el Tribunal un Recurso Directo de Nulidad, recordándole a la autoridad legislativa la importancia del respeto a la independencia judicial; cuidó celosamente la imparcialidad con la que debieron obrar los magistrados al hacer cumplir la prohibición de recibir en audiencias privadas a las partes que intervenían en un proceso constitucional; así, por ejemplo, no tuvo ningún reparo en rechazar al Alto Mando de las Fuerzas Armadas una solicitud de audiencia privada que efectuaron trasladándose personalmente hasta las oficinas del Tribunal Constitucional; recuerdo que dichas autoridades tuvieron que retornar a su sede de funciones expresando su enfado por no habérseles recibido en audiencia privada. Con su ejemplo enseñó a sus colegas y funcionarios la responsabilidad, mística y amor al trabajo".

Particularmente, considero pertinente rememorar que en el solemne acto de inicio de labores jurisdiccionales, llevado a cabo en fecha 31 de mayo de 1999, el entonces Presidente del Tribunal Constitucional de Bolivia, afirmaba enfáticamente:

"El Tribunal hablará a través de sus fallos y no hará declaraciones que no sean constitucionales. Estará atento a las inquietudes políticas y sociales que tengan relación con su labor, pero no aceptará presiones interesadas ni críticas subjetivas. Se colocará por encima de las pasiones, de las rencillas parroquiales y de los intereses subalternos. Será austero y discreto; pero reclamará el sitial que corresponde a la dignidad de la Constitución. A la legalidad de su elección tratará de agregar la legitimidad de su actuación, para merecer la confianza de las sociedades política y civil, requisito indispensable para cualquier obra de trascendencia nacional. Puesto que tenemos una misión muy alta que cumplir, nos revestiremos con la humildad del misionero; pero seremos inflexibles en el cumplimiento del deber. Y como esta misión será ardua y compleja, es posible que nos equivoquemos; pero no nos desviaremos. En otras palabras: puede que no seamos infalibles; pero seremos insospechables" 17 .

17 Cfr. Discurso del señor Presidente del Tribunal Constitucional, Dr. Pablo Dermizaky Peredo (mayo 31, Salón del Senado de la Casa de la Libertad). En: Poder Judicial de Bolivia. Labores Judiciales 1999. Sucre, Bolivia: Editorial Judicial, 2000, pp. 181-187. 
Asimismo, en cuanto a la naturaleza de las funciones del Tribunal Constitucional y la importancia de la magistratura, el Dr. Pablo Dermizaky ponía énfasis en que:

"Los Magistrados del Tribunal Constitucional somos profesionales del Derecho y, como tales, nuestro razonamiento es básicamente jurídico, sin ignorar las circunstancias políticas y sociales circundantes. Siempre que haya un conflicto inevitable entre lo jurídico y lo político, nuestro deber nos colocará del lado del Derecho. Y a quienes les cuesta aceptar la idea de que el Tribunal Constitucional puede invalidar actos del (Órgano) Legislativo, les recordamos las palabras escritas por Alexander Hamilton hace más de dos siglos: 'Esta conclusión no supone de ningún modo la superioridad del Poder Judicial sobre el Legislativo. Sólo significa que el poder del pueblo es superior a ambos, y que donde la voluntad de la legislatura, declarada en sus leyes, se halla en oposición con la del pueblo, declarada en la Constitución, los jueces deben gobernarse por la última, de preferencia a las primeras (...)"18.

Posteriormente, al culminar el primer año de labores jurisdiccionales del Tribunal Constitucional de Bolivia, el Dr. Pablo Dermizaky presentó un Informe haciendo un balance de las principales actividades realizadas por dicha institución, con especial referencia a los aspectos institucionales, jurisdiccionales y administrativos, en cuya oportunidad concluyó señalando:

"Un año es una ínfima fracción de tiempo en la vida de instituciones que, como el Tribunal Constitucional, están hechas para durar lo que dure el país como nación y como sociedad políticamente organizada. Pero si ese es el primer año, un año en el que el Tribunal ha debido conocer y resolver un mil quinientas causas, mientras continuaba organizándose, labor ésta que no ha concluido por falta de edificio propio y de recursos suficientes; un año en el que se han sentado los cimientos de una estructura institucional sólida y permanente, podemos concluir con la satisfacción del deber cumplido, con la seguridad de que hemos asumido los retos planteados por la historia y respondido con idoneidad a la misión que se nos confió, una misión que se proyecta, por ello, con una trayectoria ascendente hacia el porvenir" 19 .

18 Cfr. El Federalista. San José, Costa Rica: Edit. Libro Libre, 1986, p. 203. Citado por: Dermizaky Peredo, Pablo. La tensión entre Política y Derecho en la Justicia Constitucional. En: Tribunal Constitucional DE Bolrvia (editor). "Justicia Constitucional para comenzar el Tercer Milenio". Revista Constitucional No 3. Sucre (Bolivia): diciembre de 1999, p. 13.

19 Cfr. Poder Judicial de Bolivia. Informe del Primer Año de Labores Jurisdiccionales 1999-2000. Editorial Judicial, 2000, p. 65. 
Por último, en oportunidad de dar posesión al nuevo Presidente del Tribunal Constitucional (febrero de 2001), elegido con motivo de sus renuncias a la Magistratura y a la Presidencia del mismo, correspondió al Dr. Pablo Dermizaky hacer un breve balance de lo realizado hasta ese momento, a cuyo efecto, pronunció un breve discurso rememorando el inicio del ejercicio de las labores jurisdiccionales del Tribunal, las estadísticas existentes acerca de las causas resueltas, dando cuenta de la celeridad con que se pronunciaban las resoluciones constitucionales, para luego concluir con algunas sugerencias de modificaciones indispensables a nivel legislativo, para mejorar dicha labor. Culminó muy acongojado su discurso, con las siguientes palabras:

"Al privilegio de haber sido el primer presidente del Tribunal Constitucional sobreviene el dolor de ser el primero en abandonarlo, obligado por las circunstancias. No hay palabras para expresar la pena que me embarga desde que tomé esta ineludible decisión. Me queda el consuelo de saber que el Tribunal queda en buenas manos, con un Presidente, Magistrados y personal que desde el inicio contribuyeron a hacer de esta institución un modelo de trabajo en equipo, de eficiencia y organización"20.

Se debe mencionar también, que una vez posesionado e instalado el primer Tribunal Constitucional en Bolivia (1998-1999), recién comenzaron a escribirse y difundirse ensayos y estudios sobre la supremacía de la constitución y el control de constitucionalidad en Bolivia ${ }^{21}$, principalmente a través de la Revista

20 Cfr. Discurso del Dr. Pablo Dermizaky en la toma de posesión del nuevo Presidente del Tribunal Constitucional (Sucre, 9 de febrero de 2001). En: Poder Judicial De Bolivia. Informe del Segundo Año de Labores Jurisdiccionales junio 2000-mayo 2001. Editorial Gaviota del Sur, 2001, p. 83.

21 Y se debe anotar, que precisamente el Dr. Pablo Dermizaky Peredo y el Dr. José Antonio Rivera Santivañez (ambos Magistrados del Tribunal Constitucional), junto al Dr. Jorge Asbún (Profesor universitario), fueron los primeros autores bolivianos que en esa época, escribieron abundantemente sobre el origen, la evolución y alcances del control de constitucionalidad que se estaba implementando en Bolivia, a raíz de la reforma constitucional de 1994, dando a conocer este tema en Revistas Jurídicas especializadas a nivel nacional y latinoamericano. Cfr. Dermizaky Peredo, Pablo. "Sistemas de Control Constitucional. El Tribunal Constitucional en Bolivia". Conferencia pronunciada por el autor en un seminario auspiciado por la Universidad Mayor de San Simón y el Colegio de Abogados de Cochabamba, en octubre de 1997. En: Constitución, Democracia y Derechos Humanos. Sucre, Bolivia: Editorial Judicial, 1999, pp. 69-81. Rivera Santivañez, José Antonio. "El Control de Constitucionalidad en Bolivia”. En: Revista del Tribunal Constitucional. Número 1. Sucre, Bolivia: Editorial Judicial, 1999, pp. 45-86. Ensayo que fue publicado en el Anuario Iberoamericano de Justicia Constitucional (2003), disponible en: http://dialnet.unirioja.es/ descarga/articulo/1976174.pdf; AsBún, Jorge. "Control Constitucional en Bolivia. Evolución y Perspectivas". En: Revista Constitucional. Número 3. Sucre-Bolivia: Editorial Judicial, 1999. Ensayo publicado en el Anuario Iberoamericano de Justicia Constitucional (1999), disponible en: http://dialnet.unirioja. es/descarga/articulo/761377.pdf. 
del Tribunal Constitucional, que precisamente desde el año 1999, compilaba las ponencias y conferencias de todos los juristas nacionales e internacionales que presentaban sus investigaciones sobre la materia en los seminarios organizados por el mismo Tribunal Constitucional, y que estaban principalmente referidos a la Justicia Constitucional y su importancia en el Estado de Derecho; todo ello, dentro del Programa de Pedagogía Constitucional, un proyecto elaborado originalmente por el Dr. José Antonio Rivera Santivañez (cuando se desempeñaba como Magistrado suplente del Tribunal Constitucional), y que fue aprobado favorablemente por el Pleno del mismo Tribunal.

\section{LABOR DE PEDAGOGÍA CONSTITUCIONAL}

Sobre este aspecto, el entonces Presidente del Tribunal Constitucional de Bolivia afirmaba que las labores del Tribunal Constitucional podían agruparse en tres grandes áreas: control, interpretación y pedagogía constitucional, cuyos alcances los explicaba con bastante claridad, en el siguiente sentido:

"Interpretar la Constitución es una función que fluye naturalmente de la jurisprudencia a que da lugar el control de constitucionalidad... (de ahí que) en la importante y difícil misión que explica y legitima a un Tribunal Constitucional, su rol como intérprete último de la Constitución es, quizá, el más importante de sus trabajos. (Entonces) La interpretación del Tribunal Constitucional será una tarea constante, oportuna y activa, a diferencia de la interpretación esporádica encomendada al Órgano Legislativo por el artículo 234 de la Constitución (reformada en 1994). En cuanto a la pedagogía constitucional, es deber del Tribunal difundir el conocimiento, la comprensión y la valoración de las normas fundamentales, procesos necesarios para crear una conciencia constitucional indispensable en la construcción de una sociedad justa y progresista. (...)”. (los agregados entre paréntesis, me corresponden).

Dicho programa, que incluía conferencias, cursos y publicaciones, estaba destinado a "formar una conciencia constitucional que conduzca al conocimiento, comprensión y acatamiento de la Constitución Política del Estado, asi como de los fines y objetivos de la jurisdicción constitucional", de acuerdo a la mentalidad visionaria del entonces Presidente del Tribunal, quien era consciente de que el programa debía seguir desarrollándose, "porque constituye una de las labores concurrentes del Tribunal, sin la cual no será posible cumplir su misión principal"22.

22 Cfr. Dermizaky Peredo, Pablo. Justicia Constitucional y Estado de Derecho, pp. 11, 27 y 85. 
Cabe destacar que en varios de sus escritos, el Dr. Pablo Dermizaky resaltaba la importancia de la pedagogía constitucional entre las labores cotidianas del Tribunal Constitucional, explicando su estrecha relación con las funciones esenciales de la jurisdicción constitucional, en el siguiente sentido:

"En otras palabras, no es suficiente declarar los derechos e identificar las garantías respectivas, sino que es necesario aplicar éstas mediante un mecanismo que asegure su oportunidad y eficacia. Ese mecanismo no es otro que la jurisdicción constitucional o justicia constitucional, que es el conjunto de procedimientos destinados, según la concepción de Bidart Campos, a la defensa, control e interpretación de la Constitución.

La jurisdicción constitucional cumple, pues, una triple función, a la que nosotros hemos agregado una cuarta en el Tribunal Constitucional: la de pedagogía constitucional, que es concurrente e inseparable de las otras tres, pues, en la medida en que la jurisdicción constitucional responde a las expectativas y a las necesidades sociales, la sociedad en su conjunto aprende de ella, la aprecia y la apoya, retroalimentándola en un proceso de interacción que es vital para su mantenimiento y superación"23.

\section{APORTE A LA REFORMA CONSTITUCIONAL}

Para culminar, considero necesario poner de relieve el aporte del Dr. Pablo Dermizaky en el proceso de reforma constitucional en Bolivia ${ }^{24}$, labor a la cual fue convocado en reconocimiento de su destacada trayectoria profesional, y sobre todo por su amplio conocimiento sobre la Constitución boliviana y la necesidad de su profunda revisión, conforme había pregonado durante gran parte de su vida.

23 Cfr. Dermizaky Peredo, Pablo. Obra Citada, pp. 45-46.

24 En referencia a la adecuada configuración de las normas de procedimiento que regulan la reforma de la Constitución, el profesor Pablo Dermizaky consideraba que la clasificación que hacían algunos tratadistas de las Constituciones, en rígidas y flexibles, carecía de fundamento y habría perdido todo valor; y es que, según él, "una Constitución no debe ser ni tan rígida como un corsé, ni tan flexible como una túnica. En el primer caso se inmoviliza el cuerpo social, anquilosándolo, y en el segundo se lo deja muy suelto, sin ningún control, fuera del equilibrio a que nos referimos anteriormente. Una Constitución debe combinar en dosis adecuadas los caracteres de permanencia (que no es lo mismo que rigidez) y de cambio, que es un fenómeno constante en toda sociedad". Entonces -según el criterio de este destacado constitucionalista boliviano-, el procedimiento de la reforma constitucional no debe ser lo mismo que para las leyes ordinarias, ni uno que haga imposible, o muy difícil, la reforma. Cfr. Dermizaky Peredo, Pablo. Constitución, Democracia y Derechos Humanos, pp. 52-53. 
En efecto, mediante Resolución de la Presidencia del Congreso Nacional No 008/2000-2001, del mes de abril del año 2001, el entonces Vicepresidente de la República, dispuso aprobar la creación del Consejo Asesor de la Presidencia del Congreso Nacional, bajo la denominación de: "Consejo Ciudadano para la Reforma de la Constitución Politica del Estado", que estuvo conformado por los siguientes ciudadanos: Dr. Luis Ossio Sanjinés (Presidente), Dr. Pablo Dermizaky Peredo, Dr. Carlos Gerke Mendieta, Sr. Carlos Mesa Gisbert, Dr. Waldo Albarracín Sanchez, Lic. Martha Urioste de Aguirre, Dra. María Antonieta Pizza Bilbao, Dr. Jorge Asbún Rojas, Dr. Juan Carlos Urenda Díaz.

Este Consejo Ciudadano, tenía como principales funciones: asesorar, apoyar, promover y garantizar la participación ciudadana e institucional en la reflexión, debate y elaboración de recomendaciones que permitieran al $\mathrm{H}$. Congreso Nacional, contar con antecedentes e información útil y oportuna para efectuar la reforma de la Constitución Política del Estado. Los miembros del Consejo, fueron posesionados el día 18 de mayo de 2001, reuniéndose por vez primera de manera oficial el día 28 de mayo del mismo año. A partir de allí, iniciaron un arduo trabajo, a título honorario, por el lapso de cinco meses, con el objeto de recoger la demanda ciudadana en materia de reforma constitucional, para plasmarla en un texto ordenado y técnicamente elaborado, que expresara de la mejor manera posible, las aspiraciones de todos los bolivianos.

$\mathrm{Al}$ principio de las discusiones, se definieron los grandes temas de la reforma constitucional que el Consejo Ciudadano consideró tratar. En esa fase, se dio una profunda discusión conceptual y se llegó a los consensos generales imprescindibles para producir un texto común. Luego se inició el análisis artículo por artículo de la Constitución Política del Estado en vigencia, y la redacción de las modificaciones propuestas. Aunque el trabajo resultó moroso y difícil por la variedad de materias afrontadas, felizmente se logró culminar con la elaboración de un documento completo y de satisfacción de todos los miembros del Consejo.

El Dr. Jorge Asbún Rojas -quien fue uno de los miembros integrantes de aquel Consejo Ciudadano-, brinda un testimonio de los notables aportes del Dr. Pablo Dermizaky para consolidar la reforma constitucional, con las siguientes palabras:

"La presencia del Dr. Dermizaky en el Consejo, me permitió conocer más a fondo a la persona y debo resaltar su plena y responsable entrega al trabajo, entre los aportes que realizó merecen destacarse su sugerencias por generar una democracia más participativa y que dio lugar a la inclusión del referéndum, plebiscito e iniciativa ciudadana (art. 4 y 
40 e) y la necesidad de instaurar la Procuradoría General, cuya misión esencial sería la defensa de los intereses del Estado. (art. 131)"25.

Fue así que el Consejo Ciudadano para la Reforma Constitucional cumplió con el compromiso contraído, y el $1^{\circ}$ de noviembre del año 2001 concluyó sus labores con la redacción de la versión final de un Anteproyecto de Ley de Necesidad de la Reforma Constitucional, con la esperanza de contribuir a la consolidación de la democracia, y sobre todo al mejoramiento de las condiciones de vida de los(las) ciudadanos(as) ${ }^{26}$.

Finalmente en el año 2007, y mientras el país se encontraba en pleno proceso constituyente, la Academia Boliviana de Estudios Constitucionales (ABEC) editó un Libro colectivo en donde reunió los estudios constitucionales de diversos autores nacionales y extranjeros, en torno a un núcleo común, consistente en construir un Estado Social y Democrático de Derecho. Entre estos estudios, es posible encontrar también una última propuesta de reforma constitucional (integral), elaborada por el Dr. Pablo Dermizaky, quien justificaba el documento con las siguientes palabras:

"Hace muchos años que el autor del presente documento propuso varias reformas constitucionales, algunas de las cuales fueron adoptadas en las reformas de 1994 y 2004, como el referendo, la iniciativa popular legislativa, la Asamblea Constituyente, la abolición del monopolio partidario de la representación política, la restricción de la inmunidad parlamentaria, la introducción de la jurisdicción constitucional especializada, etc. (...) Por otra parte, el Consejo Ciudadano para la Reforma Constitucional (2001) del que el autor fue miembro, propuso al Congreso Nacional un conjunto de reformas que en su mayor parte no fueron consideradas, menos aprobadas.

El autor considera que muchas entre dichas iniciativas son válidas para una próxima reforma, y por ello las consigna en esta parte. Mención especial merece el hecho de que en el Consejo Ciudadano para la Reforma Constitucional, el autor propuso que se incluyera la Asamblea Constituyente como medio de reforma de la Constitución, propuesta que sólo fue apoyada por los doctores Waldo Albarracín

\footnotetext{
25 Entrevista efectuada por el autor al Dr. Jorge Asbún, miembro del Consejo Ciudadano para la Reforma Constitucional (vía email), el 16 de abril del 2015.

26 Cfr. Programa de Reformas Constitucionales. Anteproyecto de Ley de Necesidad de la Reforma Constitucional, elaborado por el Consejo Ciudadano para la Reforma Constitucional. La Paz, Bolivia: Editorial Offset Boliviana, 2001, pp. 7-12.
} 
y María Antonieta Pizza, por lo que, el propio Consejo no incluyó esa iniciativa en sus propuestas." 27.

Lo precedentemente expuesto, pone en evidencia la incansable lucha del Dr. Pablo Dermizaky por la adopción de una Asamblea Constituyente como mecanismo idóneo que sea previsto por la propia Constitución Política del Estado para su reforma, ello en consonancia con su ideal constante de posibilitar una participación ciudadana, efectiva y real, en la toma de decisiones políticas fundamentales para el país.

\section{EPílogo}

He ahí, algunos datos significativos que he podido indagar y sistematizar sobre la obra trascendental y el pensamiento imperecedero del profesor y jurista boliviano Pablo Dermizaky Peredo, quien ciertamente dedicó gran parte de su vida a luchar por la preservación del Estado Constitucional de Derecho y la protección de los derechos fundamentales de la persona, pregonando la importancia de estudiar el Derecho Constitucional, enseñando las virtudes y defectos de la Constitución en Bolivia, proponiendo ideas de reforma para su perfeccionamiento y adecuación a nuestra incesante evolución como sociedad, todo lo cual hizo con el único afán de lograr a través de la educación, una conciencia constitucional capaz de transformar la realidad boliviana, por medio de todos y cada uno de los(las) ciudadanos(as), para que siguiendo sus enseñanzas, seamos conscientes de nuestros derechos, pero también de nuestros deberes y obligaciones en servicio de la patria.

Por sus notables cualidades humanas, la lucidez de su visionario pensamiento, su inquebrantable firmeza en la defensa de la Constitución, su innegable convicción democrática de escuchar primero la voz del pueblo, y su inagotable labor de enseñanza académica, a través de la exposición oral o la palabra escrita, sea en la Universidad, la Academia o el Foro; Pablo Dermizaky fue, y siempre será, un boliviano imprescindible.

Ciudad del Illimani, otoño de 2015.

27 Cfr. Dermizaky Peredo, Pablo. "Propuesta de Reforma Constitucional". En: Propuestas para construir un Estado Social y Democrático de Derecho. Libro colectivo editado por la Academia Boliviana de Estudios Constitucionales. Cochabamba, Bolivia: Grupo Editorial Kipus, 2007, pp. 265-286. 